\title{
La procalcitonina come indicatore di SIRS, sepsi e shock settico
}

\author{
Roberto Rossetti, Giacomo Corsini \\ U.O. Microbiologia Spedali Riuniti Pistoia
}

Key words: procalcitonin, nosocomial infections.

\section{SUMMARY}

Nosocomial infections are an important public health problem. Procalcitonin could be used as a clinical predictor of severe nosocomial infections.

To validate procalcitonin as a diagnostic indicator of nosocomial infections, we have tested procalcitonin blood levels in 27 patients recovered in ICU of Pistoia Hospital.

LUMltest PCT® was used as a reference test.

From the comparison between low-risk and high-risk patients, selected in the considered wards, procalcitonin has emerged as a significant predictor of severe nosocomial infections.

Procalcitonin could be used, together with other common indicators, as an important and sensitive marker to differentiate systemic bacterial infections from other severe complications and to follow up patients during antibiotic treatment.

\section{INTRODUZIONE}

Le Infezioni Ospedaliere (I.O.) costituiscono tuttora una sfida importante per le molteplici implicazioni che comportano sia in campo etico e clinico, sia per i costi che esse determinano per l'aumento dei giorni di degenza e dei costi assistenziali, legati ad un maggiore impiego di farmaci e presidi diagnostici.(3) Particolare considerazione deve essere rivolta alle unità di Terapia Intensiva, dove tali patologie si presentano con maggiore frequenza e gravità ma anche altri reparti ospedalieri possono presentare le stesse problematiche, che necessitano d'interventi immediati ed il più possibile mirati per evitare esiti infausti.(7)

Per la diagnosi delle Infezioni Ospedaliere, oltre alla valutazione del quadro clinico, sono utilizzati diversi parametri di laboratorio che consentono d'inquadrare e quantificare lo stato infiammatorio specifico ma pochi sono i test in grado di monitorarne l'evoluzione e i risultati della terapia antibiotica in soggetti con infezioni batteriche sistemiche gravi.

Nel 1996 è stato introdotto, a scopo diagnostico, un nuovo indicatore biochimico, la procalcitonina (PCT) che è un propeptide ormonale della calcitonina, normalmente prodotto dalle cellule $\mathrm{C}$ della tiroide. In condizioni normali non è rilasciato in circolo mentre in corso d'infezioni batteriche gravi ne è stato evidenziato un marcato incremento ematico, che permane fino al superamento dell'evento infettivo. Molti studi hanno dimostrato che questo marcatore non è rilevabile nei casi in cui l'infezione rimane confinata ad un tessuto o ad un organo senza manifestazioni generalizzate, mentre aumenta in maniera significativa nel caso di sepsi, shock settico e in severe reazioni infiam- matorie sistemiche, fornendo quindi un mezzo per il follow-up del loro decorso clinico.

Tabella I. Valori di procalcitonina rilevabili

\begin{tabular}{lc}
\hline Pazienti & PCT (ng/ml) \\
\hline Individui normali, & \\
Processi infiammatori cronici & $<0.5$ \\
Disordini autoimmuni & \\
Infezioni virali & \\
Infezioni batteriche moderate e localizzate & \\
\hline Sindrome da risposta infiammatoria sistemica (SIRS) & $0.5-2$ \\
Traumi multipli & \\
Ustioni & $2-10$ \\
\hline Infezioni batteriche con probabile & $>10$ \\
coinvolgimento sistemico & \\
\hline Infezioni batteriche gravi & \\
Sepsi &
\end{tabular}

Al contrario, nelle infezioni virali, in corso di malattie autoimmuni, in quadri neoplastici o in corso di infezioni batteriche locali o confinate ad un organo, non si osservano significativi aumenti della procalcitonina come indicato in tabella 1.

La procalcitonina ha un'emivita in vivo di 20-24 ore ed una alta stabilità in vitro e possiede quindi i requisiti ideali per essere utilizzata come parametro giornaliero di monitoraggio. $(1,2,4,5,6,8)$

Stimolati da queste premesse abbiamo deciso di valutare l'utilità di questo marcatore su pazienti ricoverati nell'U.O. Rianimazione del nostro Ospedale utilizzando un nuovo test immunoluminometrico per la determinazione della PCT $\left(\right.$ LUMItest ${ }^{\circledR}$ PCT $(\mathrm{B} \cdot \mathrm{R} \cdot \mathrm{A} \cdot \mathrm{H} \cdot \mathrm{M} \bullet \mathrm{S}$ Diagnostica $\mathrm{GmbH}$, Berlin), introdotto sul mercato da pochi anni. 


\section{MATERIALI E METODI}

Lo studio si è svolto nel primo semestre 2002 e ha previsto l'arruolamento di tutti i ricoverati nel reparto di Rianimazione del Presidio Ospedaliero di Pistoia.

I pazienti sono stati valutati clinicamente per tutta la durata del ricovero e sono stati successivamente classificati in due gruppi: uno, di controllo, costituito da soggetti che non hanno mai presentato, durante il ricovero, segni e sintomi che potessero far diagnosticare al medico del reparto una condizione di SIRS (Systemic Inflammatory Response Syndrome o Sindrome da Risposta Infiammatoria Sistemica), sepsi o shock settico (secondo i criteri della Society of Critical Care Medicine Consensus Conference del 1992); l'altro, costituito dai soggetti in cui, almeno una volta, è stata fatta diagnosi delle condizioni cliniche sopra riportate.

Tutti i pazienti sono stati sottoposti ad un prelievo ematico effettuato al momento del ricovero e poi a giorni alterni per la valutazione della procalcitonina su siero, della proteina C-reattiva (PCR), dei globuli bianchi e delle piastrine. Le informazioni sulla situazione clinica al momento del ricovero e sul decorso durante la degenza ospedaliera sono state recuperate dalla cartella, in particolare è stato osservato l'andamento dei valori della pressione sistolica e diastolica, della frequenza cardiaca e respiratoria e della temperatura corporea.

Sono state effettuate, secondo richiesta del medico di reparto, indagini microbiologiche su lavaggio bronco alveolare (BAL), su espettorato, su sangue e su urina ed è stata annotata l'eventuale terapia antibiotica seguita dal soggetto.

Il dosaggio della procalcitonina è stato solitamente effettuato entro quattro ore dal momento del prelievo, mentre nei casi in cui ciò non era possibile e quando il prelievo era effettuato in ore di chiusura del laboratorio di analisi, si è proceduto all'immediato stoccaggio del campione di siero ad una temperatura $\mathrm{di}-20^{\circ} \mathrm{C}$ fino al momento in cui era possibile effettuare il test, di solito il giorno successivo.

Il dosaggio della procalcitonina è stato eseguito con il metodo quantitativo dopo aver ottenuto una curva di calibrazione ed impiegando $20 \mu 1$ di siero in esame. Dopo l'aggiunta del reattivo rivelatore è prodotto un segnale luminoso (RLU), la cui intensità è direttamente proporzionale alla concentrazione della procalcitonina, espressa in $\mathrm{ng} / \mathrm{ml}$.

\section{RISULTATI E DISCUSSIONE}

Sono stati arruolati 27 soggetti, 20 maschi (74\%) e 7 femmine $(26 \%)$ con una età media di 60 anni. In 17 pazienti non è mai stata fatta diagnosi clinica di SIRS, sepsi o shock settico e la PCT riscontrata in questi casi ha avuto valori compresi fra 0.08 e 2.6 di cui solo 6 hanno superato il valore soglia di $0.5 \mathrm{ng} / \mathrm{ml}$, come indicato in tabella 2 .

Tabella 2. Gruppo soggetti senza diagnosi clinica di SIRS, Sepsi Shock Settico (I 7 pazienti)

\begin{tabular}{|l|r|c|}
\hline \multicolumn{1}{|c|}{ Variabili } & Medie & Range \\
\hline Età & $\mathbf{6 4}$ & $\mathbf{1 3 - - 8 4}$ \\
\hline Degenza & 10 & $\mathbf{3 - - 6 2}$ \\
\hline Temperatura & $\mathbf{3 6 , 9}$ & $\mathbf{3 3 , 2}-\mathbf{3 9 , 8}$ \\
\hline Freq. Cardiaca & $\mathbf{9 0 , 7}$ & $\mathbf{5 3 - - 1 5 0}$ \\
\hline Globuli Bianchi & $\mathbf{1 2 . 6 2 5}$ & $\mathbf{1 . 4 4 0 - 4 4 . 0 0 0}$ \\
\hline PCR & $\mathbf{1 8 , 7}$ & $\mathbf{0 , 6}-\mathbf{1 2 4}$ \\
\hline Procalcitonina & $\mathbf{0 , 5 6 0 9}$ & $\mathbf{0 , 0 8}-\mathbf{2 , 6 0 5}$ \\
\hline
\end{tabular}

Tabella 3. Gruppo soggetti con diagnosi clinica di SIRS, Sepsi, Shock Settico ( 10 pazienti)

\begin{tabular}{|l|r|c|}
\hline \multicolumn{1}{|c|}{ Variabili } & Medie & Range \\
\hline Età & $\mathbf{5 6}$ & $\mathbf{2 1}-\mathbf{7 8}$ \\
\hline Degenza & $\mathbf{3 0}$ & $\mathbf{7 - 6 2}$ \\
\hline Temperatura & $\mathbf{3 6 , 7}$ & $\mathbf{3 4}--\mathbf{3 9 , 7}$ \\
\hline Freq. Cardiaca & $\mathbf{8 5 , 3}$ & $41--141$ \\
\hline Globuli Bianchi & $\mathbf{1 0 . 8 1 8}$ & $\mathbf{2 0 4 0 - 4 3 1 4 0}$ \\
\hline PCR & $\mathbf{1 0 , 3}$ & $\mathbf{0 , 9}-\mathbf{5 6 , 5}$ \\
\hline Procalcitonina & $\mathbf{1 2 , 1}$ & $\mathbf{0 , 0 8}-\mathbf{6 1 7 , 4}$ \\
\hline
\end{tabular}

In tabella 3 sono riportati i valori ottenuti da10 pazienti, in alcuni dei quali i quadri clinici indicati si sono verificati più volte in momenti diversi, per i quali si sono osservati 16 episodi che rientravano nei criteri stabiliti per l'inquadramento nel gruppo con diagnosi clinica di SIRS, sepsi e shock settico. In 13 casi è stato isolato un ceppo batterico presumibilmente responsabile del quadro clinico ed in alcuni casi sono stati isolati più
Tabella 4

\begin{tabular}{|l|c|c|}
\hline \multicolumn{1}{|c|}{ Batterio isolato } & Materiale & Procalcitonina \\
\hline Pseudomonas aeruginosa & BAL & 16,4 \\
\hline Branamella catarralis & BAL & 6,34 \\
\hline Streptococcus viridans & BAL & 54,7 \\
\hline Klebsiella oxytoca & BAL & 2,33 \\
\hline Pseudomonas aeruginosa & BAL & 6,5 \\
\hline Pseudomonas aeruginosa & Urina & 9,07 \\
\hline Enterococcus faecium & Sangue & 1,68 \\
\hline Streptococcus viridans & BAL & 1,65 \\
\hline Escherichia coli & BAL & 1,59 \\
\hline Corinebacterium striatum & Sangue & 0,09 \\
\hline Streptococcus bovis & BAL & 2,3 \\
\hline Haemophilus influenzae & BAL & 27,7 \\
\hline Pseudomonas aeruginosa & BAL & 1,49 \\
\hline Legionella pneumophila & Urina & BAL \\
\hline Candida albicans & BAL & BAL \\
\hline Streptococcus viridans & & \\
\hline Candida albicans & & \multirow{2}{*}{. } \\
\hline
\end{tabular}


Tabella 5

\begin{tabular}{|c|c|c|c|c|c|c|c|c|c|c|c|c|c|c|c|c|}
\hline & Giorni & Giorni & Giorni & Giorni & Giorni & Giorni & Giorni & Giorni & Giorni & Giorni & Giorni & Giorni & Giorni & Giorni & Giorni & Giorni \\
\hline Freq Cardiaca & \begin{tabular}{|l|}
86 \\
\end{tabular} & 75 & 75 & 80 & 87 & 85 & 82 & 89 & 84 & 81 & 91 & 77 & 74 & 91 & 82 & 76 \\
\hline Temp. Max & 38,5 & 37,1 & 37,1 & 37,1 & 37,6 & 36,8 & 36,7 & 36,2 & 36,3 & 38,2 & 36,4 & 36,6 & 36,2 & 37 & 36,9 & 36,9 \\
\hline Temp. Min & 36 & 36,9 & 36,7 & 36,9 & 37 & 36,6 & 36 & 36 & 36 & 36,2 & 36,1 & 35,8 & 36 & 35,8 & 36 & 36,2 \\
\hline PCR & 22,1 & 29,5 & & & 9,1 & & 20,5 & & 6,7 & & 9 & & 4,1 & & 3 & \\
\hline PCT & 617,4 & 227,16 & & & 44,98 & & 16,4 & & 7,55 & & 6,5 & & 4,6 & & 10,5 & \\
\hline Bianchi & 24910 & 23710 & 23580 & 15460 & 9000 & 9000 & 7070 & 9310 & 9020 & 10230 & 7410 & 7060 & 5760 & 3830 & 4800 & 4800 \\
\hline Emocultura & & NEG & & & & & & & & & $\mathrm{Neg}$ & & & & & \\
\hline Urinocultura & & & & & & & & & & & & & & & & \\
\hline Escreato-BAL & & & & & & & $\begin{array}{c}\text { Pseud. } \\
\text { aerugino } \\
\text { sa }\end{array}$ & & \begin{tabular}{|c|} 
Pseud. \\
Aerugino \\
sa
\end{tabular} & & & & & & & \\
\hline Antibiotico & $\begin{array}{c}\text { Tazocin } \\
2,5 g^{\star} 4 ; \\
\text { Gentalyn } \\
100 \mathrm{mg}\end{array}$ & & $\begin{array}{c}\text { Vancomic } \\
\text { ina } \\
500 \mathrm{mg} ; \\
\text { Fluconaz } \\
\text { olo } \\
200 \mathrm{mg}\end{array}$ & & & & & & $\begin{array}{c}\text { Tazocin } \\
\text { Stop; } \\
\text { Ciproxin } \\
200 \mathrm{mg}^{* 3}\end{array}$ & & $\begin{array}{c}\text { Vancocin } \\
\text { a Stop }\end{array}$ & & & & & \\
\hline
\end{tabular}

ceppi nel medesimo individuo da materiali biologici diversi.

In tabella 4 sono riportati casi in cui è stato diagnosticato un quadro di sepsi o shock settico con isolamento del batterio patogeno ed il valore di procalcitonina rilevato è stato superiore a 0,5 $\mathrm{ng} / \mathrm{ml}$ in $16 \mathrm{su} 17$ isolamenti, confermando la validità di tale marker in corso di infezioni sistemiche gravi.

In tabella 5, proposta come esempio e non per tutte le numerose giornate di ricovero, possiamo

\section{Figura I}

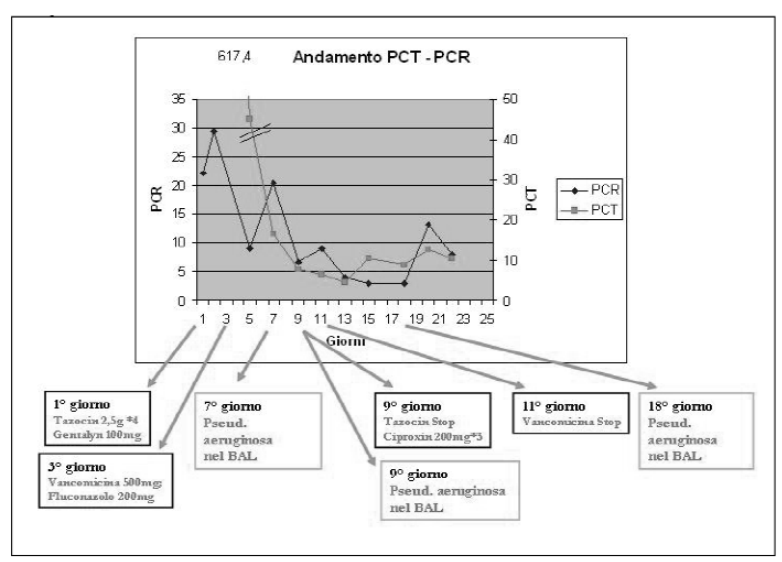

Figura II

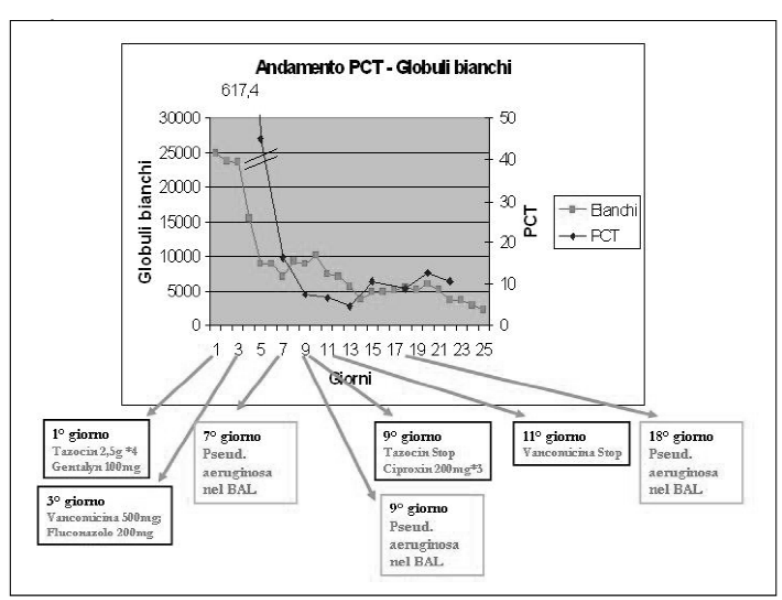

osservare in dettaglio $\mathrm{i}$ dati riportati in cartella di uno dei 10 pazienti (soggetto maschio B.R.), in cui è stato diagnosticato un grave quadro patologico infettivo.

Il paziente è stato ammesso al reparto di terapia intensiva con $38,5^{\circ} \mathrm{C}$ di temperatura, 24.910 bianchi $\mathrm{mm}^{3}$, PCR 22,1 e PCT 617,4 . E' stata iniziata una terapia antibiotica in prima giornata con Tazocin ${ }^{\circledR}$ e Gentalyn ${ }^{\circledR}$, in seconda giornata è stata effettuata una emocoltura risultata poi negativa. In terza giornata, in attesa di una risposta microbiologica, sono stati aggiunti alla terapia la vancomicina e il fluconazolo per ottenere la massima copertura possibile dai vari possibili patogeni. In settima e nona giornata è stato isolato nel BAL un ceppo di Pseudomonas aeruginosa, ma i valori di PCT erano diminuiti vistosamente già a partire dalla quarta giornata di ricovero. Questo trend aveva convinto i medici del reparto a bloccare il trattamento con il Tazocin ${ }^{\circledR}$ e, dopo due giorni, anche l'impiego della vancomicina, con il ritorno alla normalità di quasi tutti i parametri clinici e di laboratorio. Nella figura I e nella figura II è possibile osservare l'andamento della PCT molto più lineare rispetto ai valori oscillanti di PCR e globuli bianchi.

\section{CONCLUSIONI}

La nostra pur modesta esperienza si allinea ai dati presenti in letteratura sulla validità della procalcitonina come indicatore per una diagnosi precoce di SIRS, sepsi o shock settico e conferma ulteriormente la possibilità di utilizzare questo marker nel follow up di tali condizioni cliniche. Riteniamo auspicabile l'utilizzo del dosaggio della procalcitonina nella pratica clinica quotidiana nei reparti di degenza ospedaliera come ulteriore supporto laboratoristico all'attività medica per la definizione corretta e rapida del quadro clinico del paziente. 


\section{BIBLIOGRAFIA}

1. Liaudat, S; Dayer, E; Praz, G; Bille, J; Troillet, N. Usefulness of procalcitonin serum level for the diagnosis of bacteremia Eur J Clin Microbiol Infect Dis, 2001, 20 (8): 524-527.

2. Meisner M., Reinhart K. Is procalcitonin really a marker of sepsis? Int. J. Intensive Care, 2001, 8 (1): 15-25.

3. Privitera G., Panceri M.L., Castaldi S., Auxilia F. Il costo delle infezioni ospedaliere in Italia. Atti del $38^{\circ}$ Congresso Nazionale S.It.I., Fiuggi, 27-30 Settembre 1998. Ann. Ig. 10 (4 suppl. 2), 273, 1998.

4. Reinhart K. Procalcitonin as a marker of the systemic inflammatory response to infection. J Anaesth Intensivbehandlung, 2001, 2: 41-43 ${ }^{\text {b }}$

5. Reinhart, K. Procalcitonin - a new marker of severe infection and sepsis. Intensive Care Med. 2000, 26: $145^{\mathrm{a}}$.

6. Reinhart, K.; Karzai, W.; Meisner, M. Procalcitonin as a marker of the systemic inflammatory response to infection. Intensive Care Med, 2000, 26 (9): 1193-1200.

7. Vincent J.L., Bihari D.J., Suter P.M., Bruining H.A., White J. et al.,The Prevalence of Nosocomial Infection in Intensive Care Units in Europe. Results of the European Prevalence of Infection in Intensive Care (EPIC) Study. JAMA, 23-30 Agosto 1995, 274 (8).

8. Yukioka, H.; Yoshida, G.; Kurita, S.; Kato, N. Plasma procalcitonin in sepsis and organ failure Ann Acad Med Singapore. 2001, 30 (5): 528-531.

Roberto Rossetti

U.O. Microbiologia - Spedali Riuniti - ASL 3- Piazza Giovanni XXIII ${ }^{\circ}$ - 51100 PISTOIA Tel: 0573-352474/3

Fax: 0573-352474

E-mail: r.rossetti@mail.pt.usl3.toscana.it 